\title{
Three-body interacting dipolar bosons and the fate of lattice supersolidity
}

\author{
Manpreet Singh and Tapan Mishra \\ Department of Physics, Indian Institute of Technology, Guwahati-781039, Assam, India
}

(Dated: July 1, 2021)

\begin{abstract}
We investigate a system of dipolar bosons in an optical lattice with local two and three-body interactions. Using the mean-field theory approach, we obtain the ground state phase diagram of the extended Bose-Hubbard (EBH) model with both repulsive and attractive three-body interactions. We show that the additional three-body on-site interaction has strong effects on the phase diagram especially on the supersolid phase. Positive values of the three-body interaction lead to the enhancement of the gapped phases at densities larger than unity by reducing the supersolid region. However, a small attractive three-body interaction enhances the supersolid phase.
\end{abstract}

PACS numbers: 67.85.-d 67.80.kb 67.85.Hj

\section{INTRODUCTION}

The systems of ultracold quantum gases in optical lattices have been proven to be the most suitable platform for quantum simulation of complex many-body system due to the high degree of controllability of lattice parameters and geometry [1. Several interesting quantum phase transitions have been theoretically predicted and observed in the experiments in recent years. The seminal work on the quantum phase transition from the superfluid (SF) to the Mott insulator (MI) phase in dilute atomic gases 2 had paved the path to study numerous interesting quantum phenomena. The many-body physics which arises in such a dilute system is primarily attributed to the on-site two-body interaction. However, recent developments show that multi-body effects can not be ruled out as they play very significant role on the ground state properties of the system [3]. Based on the observations of collapse and revival of matter waves it has been shown that three-body interactions can play an important role in quantum phase transitions. Threeand higher-body interactions have been precisely measured for species like Rb [3] and Cs [4] in different experiments. Recent studies show that it is also possible to engineer these multi-body interactions in a controlled way in systems of ultracold atoms 5 7. One can in principle have regimes where the the signs of the two, three- and higher-body interactions can be selectively changed [5, 6].

On the other hand the long-range interaction in the polar lattice gases has been a topic of great interest in the past decade. Due to the non-local nature of the interaction, a wealth of new physics has been uncovered and one of them is the elusive supersolid (SS) phase of matter. The SS phase exhibits both superfluid and crystalline order which has attracted much attention since it was first proposed 8, 9. The presence of non-local defects on top of a crystalline order gives rise to the superfluid order in a strongly interacting system 10. It has also been predicted that supersolid may occur in lattices due to the long-range interaction. Several theoretical predictions have been made for various lattice models for the possible existence of the SS phase in the frame- work of the extended Bose-Hubbard (EBH) model [11. Also, attempts have been made to look for a scenario where the lattice supersolidity can be achieved without considering the non-local interactions in various conditions 12 14. Recent advances in the field of cooling and trapping of dipolar gases have opened up new directions to achieve longer range interaction in optical lattice 11, 15]. Other possible candidates for such strong non-local interactions are the Rydberg dressed [16, 17] atoms and polar molecules [18].

Recently the SS phase has been observed in ${ }^{87} \mathrm{Rb}$ condensate coupled with optical cavity [19, 20 where the long-range interaction is induced by the two photon processes. However, it's realisation in polar lattice gases is not yet achieved, which promises to be the most suitable system. Experimental progress in loading polar lattice gases in optical lattices [21, 22] and most recently the realisation of the $\mathrm{EBH}$ model by loading dipolar gas of ${ }^{168} \mathrm{Er}$ atoms in a three-dimensional optical lattice [23], have paved the path to observe the exotic SS phase of matter.

Although the SS phase can be achieved only in the presence of the on-site and nearest neighbour two body interactions, three-body interactions play very important role in deciding the fate of the supersolid phase. The effects of dominant two- and three-body local interactions in ultracold atomic systems have been studied extensively 2433 . It has also been shown that the cold polar molecules can interact via a long-range three-body interaction as well 34 , 38 , leading to interesting quantum phenomena. However, the effect of local three-body interaction on the phase diagram of the EBH model, especially on the supersolid phase has not been explored in detail.

In this paper we consider a system of dipolar bosons with both on-site, two- and three-body interactions in an optical lattice, which can be explained by a modified $\mathrm{EBH}$ model. We analyse the ground state properties for both positive and negative three-body interactions with different combinations of the on-site and nearest neighbour repulsion. In our study, we show that the three-body interaction has very significant effect on the phase diagram of the EBH model. At incommensurate 
densities, repulsive three-body interaction enhances the gapped phases and simultaneously shrinks the SS region. However, the physics of the system becomes interesting when we consider a very small and attractive three-body interaction. In this case the supersolid phase becomes more robust. The SS region in the phase diagram gets enhanced by pushing the SS-SF boundary towards large hopping values. The three-body on-site interaction also introduces other gapped phases at commensurate densities due to the breaking of the degeneracies.

The remaining part of the paper in organised as follows. In Sec. II, we give details of the model and the method used in this work. Section III contains the discussion about the results and we conclude in Sec. IV.

\section{MODEL AND METHOD}

The effective many-body Hamiltonian which describes the system considered here is given by

$$
\begin{aligned}
H= & -t \sum_{<i, j>}\left(a_{i}^{\dagger} a_{j}+H . c .\right)+\frac{U}{2} \sum_{i} n_{i}\left(n_{i}-1\right) \\
& +V \sum_{<i, j>} n_{i} n_{j}+\frac{W}{6} \sum_{i} n_{i}\left(n_{i}-1\right)\left(n_{i}-2\right) \\
& -\mu \sum_{i} n_{i}
\end{aligned}
$$

where $a_{i}^{\dagger}\left(a_{i}\right)$ are the bosonic creation (annihilation) operators at the site $i, t$ is the hopping amplitude between the adjacent sites $\langle i, j\rangle, n_{i}$ is the number operator at site $i$. $U$ and $W$ are the on-site two- and three-body interactions, $V$ is the nearest neighbour interaction (NNI) between the particles located at sites $i$ and $j$, and $\mu$ is the chemical potential. We investigate the ground state properties of the model (1) using the single site mean-field decoupling approximation [39]. This method has been widely used to investigate the quantum phase transitions in systems of ultracold quantum gases in optical lattices. Although this method is more accurate for higher dimensions and large densities, where quantum fluctuations become less dominant, it gives enough information to qualitatively understand the underlying physics of the system in low dimensional cases. It is to be noted that in the case of dipolar atoms with repulsive $V$ in optical lattices, 1D and $2 \mathrm{D}$ systems are more relevant from the experimental point of view. However, in order to understand an overall picture we investigate the global phase diagram for a d-dimensional lattice.

In the present case, using the MF decoupling approximation one can make the following substitutions,

$$
\begin{aligned}
a_{i}^{\dagger} a_{j} & \simeq\left\langle a_{i}^{\dagger}\right\rangle a_{j}+a_{i}^{\dagger}\left\langle a_{j}\right\rangle-\left\langle a_{i}^{\dagger}\right\rangle\left\langle a_{j}\right\rangle \\
n_{i}^{\dagger} n_{j} & \simeq\left\langle n_{i}^{\dagger}\right\rangle n_{j}+n_{i}^{\dagger}\left\langle n_{j}\right\rangle-\left\langle n_{i}{ }^{\dagger}\right\rangle\left\langle n_{j}\right\rangle
\end{aligned}
$$

in Eq1 to write the single site MF Hamiltonian as:

$$
\begin{aligned}
\frac{H_{i}^{M F}}{z t} & \equiv \frac{1}{2} \frac{U}{z t} n_{i}\left(n_{i}-1\right)-\frac{\mu}{z t} n_{i}-\left(\phi_{i} a_{i}^{\dagger}+\phi_{i}^{*} a_{i}\right) \\
& +\frac{1}{2}\left(\psi_{i}^{*} \phi_{i}+\psi_{i} \phi_{i}^{*}\right)+\frac{V}{t}\left(n_{i} \bar{\rho}_{i}-\rho_{i} \bar{\rho}_{i}\right) \\
& +\frac{1}{6} \frac{W}{z t} n_{i}\left(n_{i}-1\right)\left(n_{i}-2\right),
\end{aligned}
$$

where $\psi_{i} \equiv\left\langle a_{i}^{\dagger}\right\rangle \equiv\left\langle a_{i}\right\rangle$ and $\rho_{i} \equiv\left\langle n_{i}\right\rangle$ are the superfluid order parameter and density, respectively, $z(=2 d)$ is the co-ordination number of a site in a $d$-dimensional hypercubic lattice, and $\phi_{i} \equiv \frac{1}{z} \sum_{i^{\prime}} \psi_{i+i^{\prime}}, \bar{\rho}_{i} \equiv \frac{1}{z} \sum_{i^{\prime}} \rho_{i+i^{\prime}}$; $i^{\prime}$ takes into account the nearest-neighbours given by $z$. Due to the possible presence of the DW order in the ground state, we consider a bipartite lattice configuration. We denote these sublattices by $\alpha$ and $\beta$ and write the MF Hamiltonian as the sum of the Hamiltonians for these two sublattices.

$$
H^{M F}=H_{\alpha}^{M F}+H_{\beta}^{M F}
$$

The $\phi$ and $\bar{\rho}$ terms in Eq 3 contain contribution from the neighbouring sites, that is, if $i=\alpha, \phi$ and $\bar{\rho}$ contain contribution from $\beta$-sublattice and vice versa. Therefore, we can write

$$
\begin{aligned}
\frac{H_{\alpha}^{M F}}{z t} & \equiv \frac{1}{2} \frac{U}{z t} n_{\alpha}\left(n_{\alpha}-1\right)-\frac{\mu}{z t} n_{\alpha}-\left(\psi_{\beta} a_{\alpha}^{\dagger}+\psi_{\beta}^{*} a_{\alpha}\right) \\
& +\frac{1}{2}\left(\psi_{\alpha}^{*} \psi_{\beta}+\psi_{\alpha} \psi_{\beta}^{*}\right)+\frac{V}{t}\left(n_{\alpha} \rho_{\beta}-\rho_{\alpha} \rho_{\beta}\right) \\
& +\frac{1}{6} \frac{W}{z t} n_{\alpha}\left(n_{\alpha}-1\right)\left(n_{\alpha}-2\right),
\end{aligned}
$$

Similarly, we obtain the expression for $H_{\beta}^{M F}$. We then construct the Hamiltonian matrix for Eq,4 in the occupation number basis and diagonalize it self-consistently to obtain the ground state energy and wavefunction. The ground-state wavefunction is then used to calculate the superfluid density $\left(\rho_{s}=\phi^{2}\right)$ and number density $(\rho)$ of the system. The maximum occupancy we considered for our calculations is 9 atoms per site. Hereafter we use $\operatorname{bar}(-)$ to denote the scaling by $z t$, e.g., $\bar{U}=U / z t$.

\section{RESULTS AND DISCUSSION}

The phase diagram of the EBH model in the absence of $W$ is studied extensively in the recent years [11. In the limit of negligible hopping and $z \bar{V}<\bar{U}$ the system exhibits alternate gapped DW and MI phases at commensurate densities. In the DW phases the alternate sites are occupied by the same number of particles due to the nearest-neighbour dipole-dipole repulsion. However, in the MI phase particle distribution is uniform throughout the lattice. On the other hand when $z \bar{V}>\bar{U}$, there exists only the DW phases. In both the limits, when the hopping amplitude $t$ increases, the SS phase starts to appear around the DW phases and further increasing the 
value of $t$ leads to the SF phase. Recent mean-field theory calculation using Gutzwiller approach predicts the above findings in the case of a d-dimensional hypercubic lattice [40. It has also been shown that when the value of $\bar{V}$ increases, the SS region gets enlarged by pushing the SS-SF boundary. Similar studies in 1D, 2D and 3D systems have been carried out using powerful methods like the quantum Monte Carlo (QMC) and the density matrix renormalization group (DMRG) methods 41-45.

Using our mean-field calculation we first obtain the ground state phase diagram of the EBH model (with $\bar{W}=0$ ) for two regions of the NNI, one below the critical point $z \bar{V}<\bar{U}(z \bar{V}=0.9 \bar{U})$ and other above the critical point $z \bar{V}>\bar{U}(z \bar{V}=1.5 \bar{U})$, as already discussed in Ref. [40. Then we analyse the effect of both repulsive and attractive three-body interactions $\bar{W}$. Our main findings are depicted in Fig. 22 and 3. Before proceeding further we give the details of the abbreviations used for the gapped phases in the following table.

\begin{tabular}{|c|c|c|}
\hline Phase & Configuration & Density $\rho$ \\
\hline MI1 & $\begin{array}{lllll}1 & 1 & 1 & 1 & 1\end{array}$ & 1.0 \\
\hline MI2 & 22222 & 2.0 \\
\hline DW1 & $\begin{array}{lllll}1 & 0 & 1 & 0 & 1\end{array}$ & 0.5 \\
\hline DW2 & 2002002 & 1.0 \\
\hline DW3 & 21212 & 1.5 \\
\hline DW4 & 300303 & 1.5 \\
\hline DW5 & $\begin{array}{lllll}3 & 1 & 3 & 1 & 3\end{array}$ & 2.0 \\
\hline DW6 & 40404 & 2.0 \\
\hline DW7 & 32323 & 2.5 \\
\hline
\end{tabular}

TABLE I. Density configuration of gapped MI and DW phases at commensurate densities.

We use the average superfluid density $\rho_{s, a v g}\left(=\left(\rho_{s, \alpha}+\right.\right.$ $\left.\left.\rho_{s, \beta}\right)\right) / 2$ and average sublattice density $\rho_{\text {avg }}\left(=\left(\rho_{\alpha}+\right.\right.$ $\left.\left.\rho_{\beta}\right)\right) / 2$ as a function of chemical potential to distinguish the gapped phases from the gapless phases. In the gapped phases $\rho_{s}$ vanishes and there appears a plateau in the chemical potential $\bar{\mu}$. The DW phases can be distinguished by the finite density imbalance between the $\alpha$ and $\beta$ sublattices. The SS phase is identified by looking for finite superfluid density and finite density imbalance as a function of the chemical potential.

In the absence of the three-body interaction $\bar{W}$, as predicted before in Ref. [40, for $z \bar{V}=0.9 \bar{U}$ the system exhibits alternate gapped MI and DW phases at commensurate densities which is shown by plotting the densities with respect to the chemical potential in Fig.1(a). We plot $\rho_{\alpha}$ (red dashed line), $\rho_{\beta}$ (green dot-dashed line), $\rho_{\text {avg }}$ (blue solid line) and $\rho_{s, a v g}$ (magenta circles) with respect to $\bar{\mu}$ for $\bar{U}=15.0$ and $z \bar{V}=0.9 \bar{U}$. It can be seen that the plateaus appear at $\rho_{\text {avg }}=0.5,1.0,1.5,2.0,2.5$ corresponding to the gapped phases DW1, MI1, DW3, MI2 and DW7 phases, respectively. The associated arrangement of atoms in sublattices $\alpha$ and $\beta$ are shown in the Table I. In the plateau regions the superfluid density

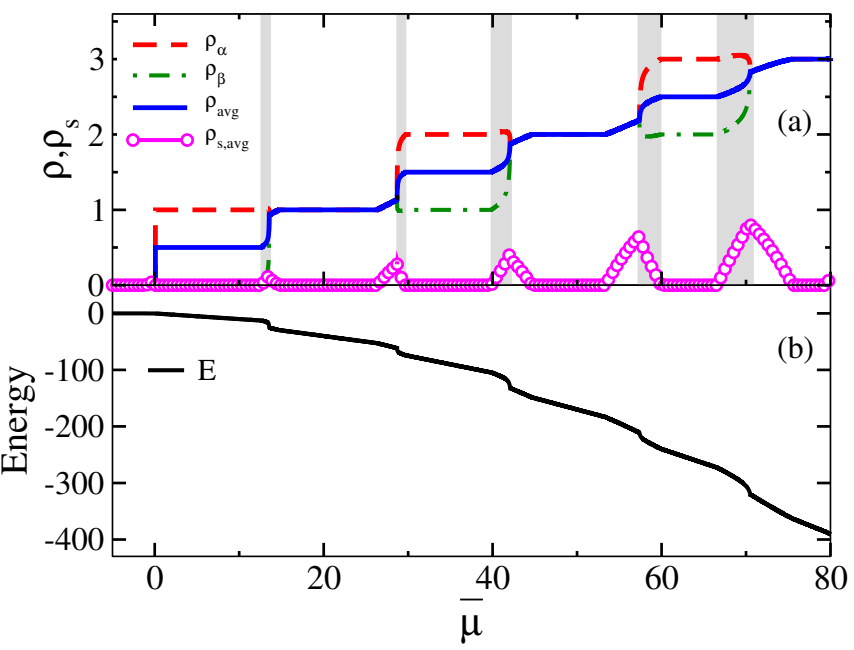

FIG. 1. (Color online) (a)The sublattice densities $\rho_{\alpha, \beta}$, the average density $\rho_{a v g}$ and the average superfluid density $\rho_{s, a v g}$, as a function of the chemical potential $\bar{\mu}$ for $\bar{U}=15.0$, $z \bar{V}=0.9 \bar{U}$ and $\bar{W}=0$. The plateaus at commensurate densities show the gapped phases where $\rho_{s}$ vanishes. The density imbalance on two sublattices shows the signature of the DW order. The SS phases are marked by grey shaded regions. (b) The ground state energy plotted with respect to $\bar{\mu}$ showing a clear change in slope at the SF-SS transitions. The slope changes at other phase transitions also (not clearly visible in the present scale of the axes).

$\rho_{s, a v g}$ vanishes. The DW phases are identified by looking at the sublattice densities $\rho_{\alpha}$ and $\rho_{\beta}$. The region around the plateaus are gapless SF phases. However, there are certain regions where there exists finite sublattice density imbalance in the SF regions. These regions correspond to the SS phase as discussed before.

By repeating the calculation for different values of $\bar{U}$ we obtain the complete phase diagram of the model given in Eq. 11) for $\bar{W}=0$ (Fig2(a), previously shown in Ref. 40,) and two different values of $\bar{W}=0.5 \bar{U}, 1.0 \bar{U}$ in Fig 2(b), (c), respectively. The phase diagrams are obtained by locating the end points of the plateaus for the gapped phases and for the supersolid phase we look for the points where the density imbalance disappears inside the SF phase. In Fig 2(a), for $\bar{W}=0$, the gapped MI and DW lobes are formed in the strong interaction regime. In the weak interaction regime the SF phase appears and the DW lobes are surrounded by the SS phases. In our analysis we truncate the phase diagram at the DW lobe of density 2.5 as it is sufficient for the relevant physics.

\section{A. Repulsive 3-body interaction}

By turning on the three-body interaction $\bar{W}$ we see a clear effect on the quantum phases at large densities as shown in the phase diagrams. In Fig 2(b) and (c) it can be seen that the gapped DW3, MI2 and DW7 lobes become larger and the SS phases shrink drastically as $\bar{W}$ 


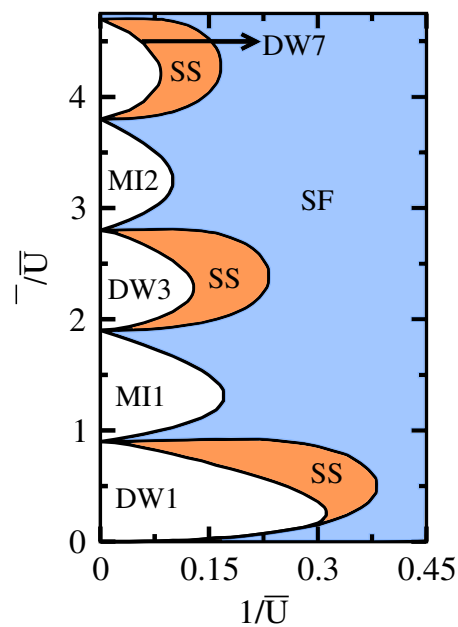

(a)

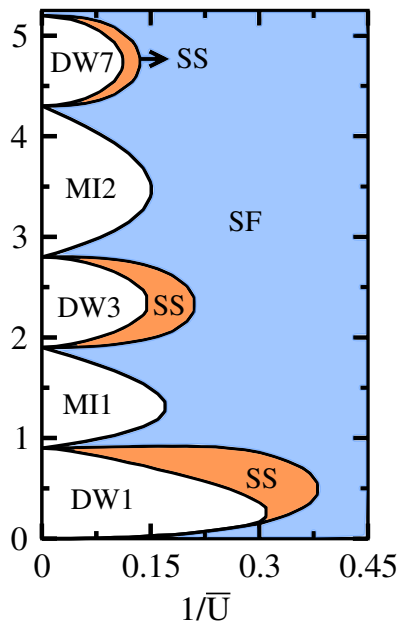

(b)

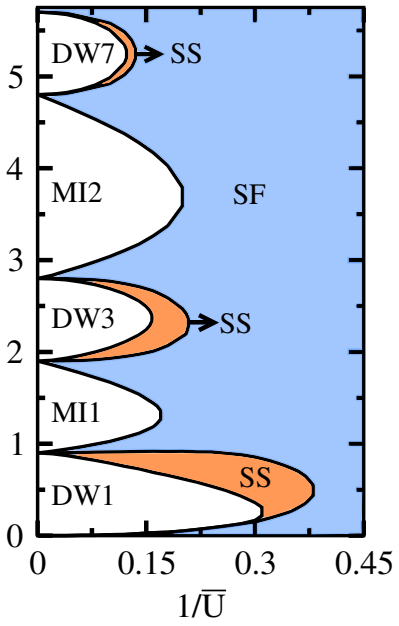

(c)

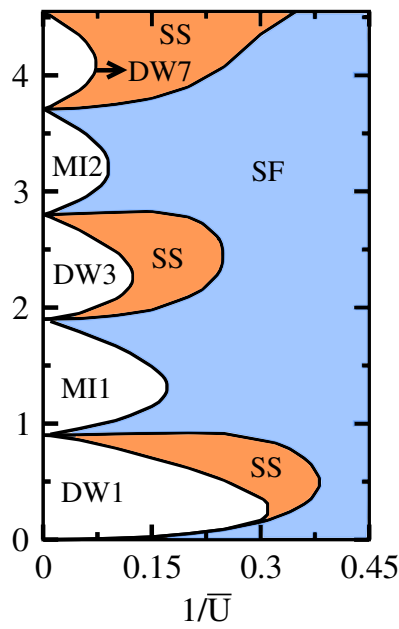

(d)

FIG. 2. (Color online) Phase diagram of model 11 with repulsive and attractive three-body interaction $\bar{W}$ for $z \bar{V}=0.9 \bar{U}$. The values of $\bar{W}$ are (a) $\bar{W}=0$, (b) $\bar{W}=0.5 \bar{U},(\mathrm{c}) \bar{W}=1.0 \bar{U}$ and (d) $\bar{W}=-0.09 \bar{U}$.

increases. This can be understood as follows: although the range of $\bar{U}$ and $\bar{V}$ is fixed, the positive values of $\bar{W}$ increase the effective onsite interaction. As a result the gapped phases lobes, such as that of the MI and DW, increase in size and the SS region shrinks. We repeat the same calculation but with $z \bar{V}=1.5 \bar{U}$ and show the result in Fig $3(\mathrm{a})$-(c). In the absence of $\bar{W}$, as predicted in Ref. 40, the MI phases disappear and only the DW phases such as DW1, DW2, DW4 and DW6 appear as shown in Fig 3(a). The SS phase in this case occupies a large region of the phase diagram. The effect of repulsive three-body interaction can be seen in the phase diagrams given in Fig, 3 (b) and Fig 3 (c). For $\bar{W}=0.5 \bar{U}$ the size of DW2 and DW4 lobes increases, whereas the DW6 phase disappears due to the effect of the repulsive $W$ as shown in Fig 3(b). At the same time DW5 phase appears in the phase diagram. The SS phase shrinks drastically similar to the case for $z \bar{V}<\bar{U}$. The SS-SF boundary in this case seems to close after the DW5 lobe unlike the case for $\bar{W}=0$ where the SS-SF boundary grows almost linearly. When $\bar{W}$ is increased further i.e. for $\bar{W}=1.0 \bar{U}$ the on-site repulsion becomes more dominant and hence the system does not allow individual lattice sites to have more than two atoms. This leads to the disappearance of the DW4 phase and appearance of the DW3 phase. Surprisingly, the competition between $\bar{W}$ and $\bar{V}$ leads to the appearance of MI2 phase which is absent with only local two-body interaction. The SS phase shrinks and envelopes all the DW phases as shown in Fig 3 (c). The SF-DW phase transition below $\rho=0.5$ is a first order transition, where as the SS-SF transition and DW-SS transitions are found to be continuous [46. The signatures of these phase transitions can be seen from the $\rho$ vs. $\mu$ plot as shown in Fig,1(a). The corresponding ground state energy with respect to $\bar{\mu}$ also shows the change in slope at different phase transitions points as shown in Fig 1(b).

\section{B. Attractive 3-body interaction}

In this section we discuss the effect of attractive threebody interaction. It is well known that a small attractive local interaction leads to the collapse of atoms onto a single lattice site [47. However, due to the presence of two-body repulsion, the system does not collapse up to a certain threshold value of $\bar{W}$. For the situation considered here, i.e. for $z \bar{V}=0.9 \bar{U}$ the collapse occurs at $\bar{W}=-0.21 \bar{U}$. Hence, in this case we restrict ourselves to very small negative values of $\bar{W}$ compared to the repulsive two-body interaction $\bar{U}$. We find that though $\bar{W}$ is very small, it has a prominent effect on the phase diagram. Interestingly, the attractive three-body interaction favours the SS phase which can be seen from Fig. 2(d) and Fig. 3(d) for $z \bar{V}=0.9 \bar{U}, \bar{W}=-0.09 \bar{U}$ and $z \bar{V}=1.5 \bar{U}, \bar{W}=-0.1 \bar{U}$ respectively. It can be seen that when $z \bar{V}<\bar{U}(z \bar{V}=0.9 \bar{U})$, there is a small reduction in the DW3 and DW7 lobes (Fig. 2(d)) as compared to the case of $\bar{W}=0$ (Fig. 2(a)). The tip of the DW3 phase shifts from $1 / \bar{U}=0.13$ to 0.12 where as the tip of DW7 phase shifts from $1 / \bar{U}=0.84$ to 0.73 . This reduction is obvious as the attractive three-body interaction reduces the effective on-site interaction by a small amount. This in turn reduces the gapped phases at higher densities as predicted in Ref. 25 for the Bose-Hubbard model.

However, the competition between the strong repulsive $\bar{U}, \bar{V}$ and weak attractive $\bar{W}$ modifies the SS phase drastically. The SS regions enlarge in the vicinity of the DW phases and the SS-SF boundary shifts towards the weak coupling regimes as shown in Fig. 2(d). By comparing Fig. 2(a) and (d), it can be seen that the tip of the SS region (surrounding the DW3 lobe) shifts from $1 / \bar{U} \approx 0.23$ to 0.25 . The width of SS region also increases, e.g. at $1 / \bar{U}=0.2,(\mu / U)_{\min }$ changes from 2.1 to 2.09 and $(\mu / U)_{\max }$ changes from 2.68 to 2.78 . Here $(\bar{\mu} / \bar{U})_{\min }$ and $(\bar{\mu} / \bar{U})_{\max }$ are the points on the lower and 


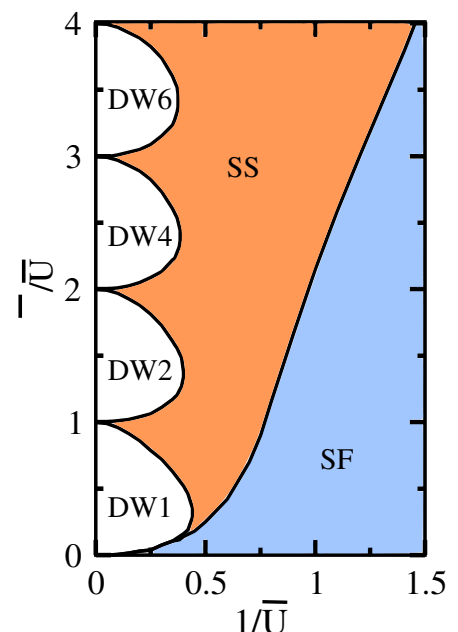

(a)

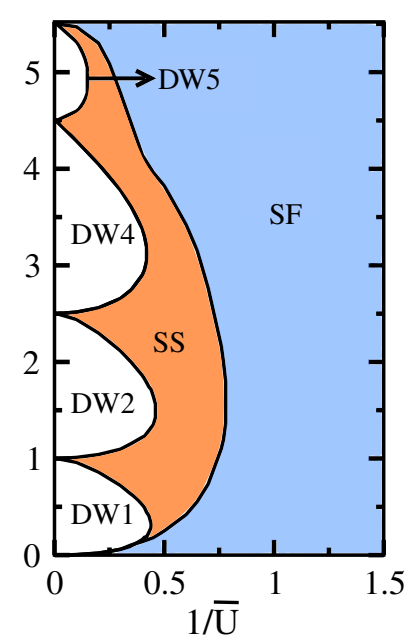

(b)

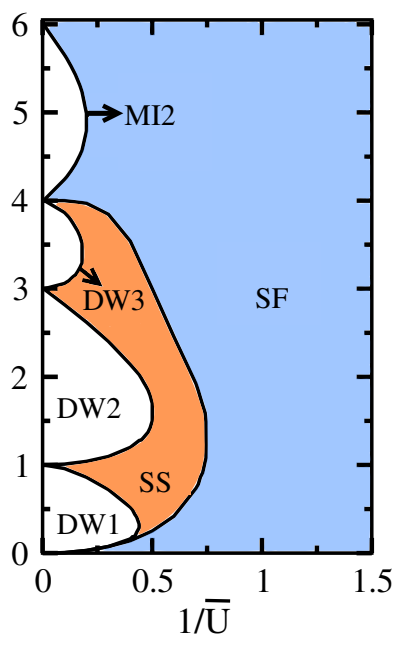

(c)

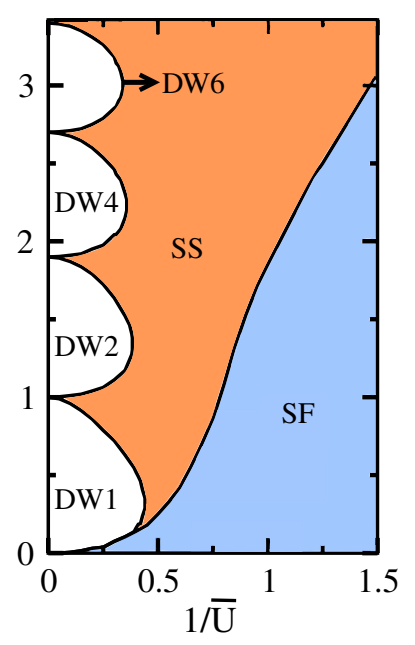

(d)

FIG. 3. (Color online) Phase diagram of model $\sqrt{1}$ with repulsive and attractive three-body interaction $\bar{W}$ for $z \bar{V}=1.5 \bar{U}$. The values of $\bar{W}$ are (a) $\bar{W}=0$, (b) $\bar{W}=0.5 \bar{U},(\mathrm{c}) \bar{W}=1.0 \bar{U}$ and (d) $\bar{W}=-0.1 \bar{U}$.

upper boundary of the SS region, respectively. This effect is more prominent for larger densities as seen in the region around the DW7 phase as shown in Fig. 2(d). The enhancement of the SS phase is very similar to the case when $z \bar{V}>\bar{U}$ and $\bar{W}=0$ as predicted in Ref. [40]. This finding shows that a small negative three-body interaction can actually play a very important role in enlarging the SS phase even without making the dipole-dipole interaction $z \bar{V}$ stronger than the on-site interaction $\bar{U}$ at large densities, which is a relevant experimental condition. For the case of $z \bar{V}=1.5 \bar{U}$ and $\bar{W}=-0.1 \bar{U}$, similar effects are seen. The DW phases at higher densities such as the DW2, DW4 and DW6 phases shrink and there is a clear enhancement of the SS phase in the vicinity of the DW4 and DW6 phases which can be seen by comparing Fig 3 (a) and Fig 3 (d). For example, at $1 / \bar{U}=1.0$, $(\bar{\mu} / \bar{U})_{\text {min }}$ changes from 2.15 to 1.85 and at $1 / \bar{U}=1.5$ it changes from 4.18 to 3.06 . The enhancement of the SS phase can be attributed to reduction in the effective on-site repulsion due to the attractive three-body interaction while the nearest neighbour interaction remains unaffected. As a result the defects in the system become more mobile on top of the DW phase and hence the supersolid nature increases. Therefore, the SS-SF phase boundary shifts towards the large values of $1 / \bar{U}$. It is to be noted that the system gradually collapses by making $\bar{W}$ further attractive.

\section{CONCLUSIONS}

We investigate a system of dipolar bosons in a $d$ dimensional lattice in the framework of the modified EBH model and study the effect of the three-body local interaction on the supersolid phase using the self consistent mean-field decoupling approximation. Considering two limiting cases such as $z \bar{V}=0.9 \bar{U}$ and $z \bar{V}=1.5 \bar{U}$, we show that the phase diagram changes drastically due to the effect of both repulsive and attractive three-body interactions. In the case of repulsive $\bar{W}$, the lobes corresponding to the gapped phases such as the MI and the DW phase enlarge at densities larger than unity. As a result the SS phase shrinks substantially. When $z \bar{V}>\bar{U}$, new DW phases appear due to the breaking of the degeneracies. Also the MI phase reappears when $\bar{W}=1.0 \bar{U}$ which was not the case when $\bar{W}=0$. In this case the SS phase tends to shrink and surround multiple DW lobes in contrast to the case of $\bar{W}=0$ where the SS phase expands pushing the SS-SF boundary almost linearly [40. However, the situation is completely different when a very small attractive three-body interaction is introduced. Interestingly, the small attractive $\bar{W}$ favours the SS phase which enlarges further as compared to the $\bar{W}=0$ case.

As discussed before, the effects of three-body on-site interaction in ultracold atomic systems are generally considered to be small. However, recent progress in theoretical studies show that the three-body interaction can be engineered under proper conditions [5], 48. Threebody interaction can also be made negative keeping the two-body interaction positive [5, 6, 48. In principle, it is possible to tune the two and three-body interactions independently with respect to the off-site dipole-dipole interaction. Hence, our findings provide further scopes to observe the effect of the local three-body interaction on the phase diagram of the EBH model and in particular the exotic supersolid phase of matter using tunable attractive three-body interaction at large densities. 


\section{ACKNOWLEDGEMENT}

We would like to thank Luis Santos, Paolo Pedri, Subroto Mukerjee, Ramesh V. Pai, Sebastian Greschner and Sebastian Will for the useful discussions. MS would like to acknowledge support from DST-SERB, India for the financial support through project number PDF/2016/000569. TM acknowledges the support by the start-up research grant from the Indian Institute of Technology, Guwahati, India. Computational work was done using the HPC (Param Ishan) facility at the Indian Institute of Technology, Guwahati.
[1] M. Lewenstein, A. Sanpera, V. Ahufinger, Ultracold Atoms in Optical Lattices, (Oxford Univ. Press), (2012).

[2] M. Greiner, O. Mandel, T. Esslinger, T. W. Hänsch, and I. Bloch, Nature 415, 39 (2002).

[3] S. Will, T. Best, U. Schneider, L. Hack-ermüller, DirkSören Lühmann, and I. Bloch, Nature 465, 197 (2010).

[4] M. J. Mark, E. Haller, K. Lauber, J. G. Danzl, A. J. Daley, and H.-C. Nägerl, Phys. Rev. Lett. 107, 175301 (2011).

[5] D. S. Petrov, Phys. Rev. Lett 112, 103201 (2014).

[6] D. S. Petrov, Phys. Rev. A 90, 021601 (2014).

[7] A. J. Daley and J. Simon, Phys. Rev. A 89, 053619 (2014).

[8] A. F. Andreev and I. M. Lifshitz, Sov. Phys. JETP 29, 1107 (1969).

[9] G. V. Chester, Phys. Rev. A 2, 256 (1970).

[10] M. Boninsegni, and N. Prokofev, Rev. Mod. Phys. 84, 759 (2012).

[11] M. A. Baranov, M. Dalmonte, G. Pupillo, and P. Zoller, Chem. Rev. 112, 5012 (2012), and references therein.

[12] S. Takayoshi, H. Katsura, N. Watanabe, and H. Aoki, Phys. Rev. A 88, 063613 (2013).

[13] M. Tovmasyan, E. P. L. van Nieuwenburg, and S. D. Huber, Phys. Rev. B 88, 220510(R) (2013).

[14] T. Mishra, S. Greschner, and L. Santos, Phys. Rev. B 92, 195149 (2015).

[15] T. Lahaye, C. Menotti, L. Santos, M. Lewenstein, and T. Pfau, Rep. Prog. Phys., 72, 126401 (2009).

[16] T. F. Gallagher and P. Pillet, Advances In Atomic, Molecular, and Optical Physics, Vol. 56, edited by E. Arimondo, P. R. Berman, and C. C. Lin (Academic Press, London, 2008), p. 161.

[17] F. Cinti, T. Macrì, W. Lechner, G. Pupillo, and T. Pohl, Nat. Commun. 5, 3235 (2014).

[18] S. Ospelkaus, K.-K. Ni, D. Wang, M. H. G. de Miranda, B. Neyenhuis, G. Quéméner, P. S. Julienne, J. L. Bohn, D. S. Jin, and J. Ye, Science 327, 853 (2010).

[19] K. Baumann, C. Guerlin, F. Brennecke and T. Esslinger, Nature 464, 1301 (2010).

[20] R. Landig, L. Hruby, N. Dogra, M. Landini, R. Mottl, T. Donner, and T. Esslinger, Nature 532, 476 (2016).

[21] J. Stuhler, A. Griesmaier, T. Koch, M. Fattori, T. Pfau, S. Giovanazzi, P. Pedri, and L. Santos, Phys. Rev. Lett. 95, 150406 (2005).

[22] T. Koch et al., Nature Phys. 4, 218 (2008).

[23] S. Baier, M. J. Mark, D. Petter, K. Aikawa, L. Chomaz, Z. Cai, M. Baranov, P. Zoller, and F. Ferlaino, Science 352, 201 (2016).

[24] M. Singh, A. Dhar, T. Mishra, R. V. Pai, and B. P. Das, Phys. Rev. A 85, 051604(R) (2012).
[25] T. Sowiński, Phys. Rev. A 85, 065601 (2012).

[26] T. Sowiński, R. W. Chhajlany, O. Dutta, L. Tagliacozzo, and M. Lewenstein, Phys. Rev. A 92, 043615 (2015).

[27] Bo-lun Chen, Xiao-bin Huang, Su-peng Kou, and Y. Zhang, Phys. Rev. A 78, 043603 (2008).

[28] W. Zhang, Ran Li, W. X. Zhang, C. B. Duan, and T. C. Scott, Phys. Rev. A 90, 033622 (2014); W. Zhang, Y. Yang, L. Guo, C. Ding, and T. C. Scott, Phys. Rev. A 91, 033613 (2015).

[29] S. Greschner, L. Santos, and T. Vekua, Phys. Rev. A 87, 033609 (2013).

[30] J. Silva-Valencia and A. M. C. Souza, Phys. Rev. A 84, 065601 (2011).

[31] Yu-Chun Chen, Roger G. Melko, S. Wessel, and Ying-Jer Kao, Phys. Rev. B 77, 014524 (2008).

[32] S. Ejima, F. Lange, H. Fehske, F. Gebhard, and K. zu Münster, Phys. Rev. A 88, 063625 (2013).

[33] C.A. Avila, R. Franco, A.M.C. Souza, M.S. Figueira, and J. Silva-Valencia, Phys. Lett. A 378, 3233 (2014).

[34] H. P. Büchler, A. Micheli, and P. Zoller, Nature Phys. 3, 726 (2007).

[35] Kai P. Schmidt, J. Dorier, and Andreas M. Läuchli, Phys. Rev. Lett. 101, 150405 (2008).

[36] B. Capogrosso-Sansone, S. Wessel, H. P. Büchler, P. Zoller, and G. Pupillo, Phys. Rev. B 79 020503(R) (2009).

[37] L. Bonnes and S. Wessel, Phys. Rev. B 83, 134511 (2011).

[38] T. Mishra, S. Greschner, and L. Santos, Phys. Rev. A 91, 043614 (2015).

[39] K. Sheshadri, H. R. Krishnamurthy, R. Pandit, and T. V. Ramakrishnan, Europhys. Lett. 22, 257 (1993).

[40] M. Iskin, Phys. Rev. A 83, 051606(R) (2011).

[41] T. Mishra, Ramesh V. Pai, S. Ramanan, Meetu Sethi Luthra, and B. P. Das, Phys. Rev. A 80, 043614 (2009).

[42] G. G. Batrouni, R. T. Scalettar, G. T. Zimanyi, and A. P. Kampf, Phys. Rev. Lett. 74, 2527 (1995).

[43] P. Sengupta, L. P. Pryadko, F. Alet, M. Troyer, and Guido Schmid, Phys. Rev. Lett. 94, 207202 (2005).

[44] T. Ohgoe, T. Suzuki, and N. Kawashima, Phys. Rev. Lett. 108, 185302 (2012).

[45] Bin Xi, Fei Ye, W. Chen, F. Zhang, and Gang Su, Phys. Rev. B 84, 054512 (2011).

[46] D. L. Kovrizhin, G. V. Pai, and S. Sinha, Europhys. Lett. 72, 162 (2005).

[47] F. Dalfovo, S. Giorgini, L. P. Pitaevski, and S. Stringari, Rev. Mod. Phys. 71, 463 (1999).

[48] P. R. Johnson, E. Tiesinga, J. V. Porto, and C. J. Williams, New J. Phys. 11, 093022 (2009). 